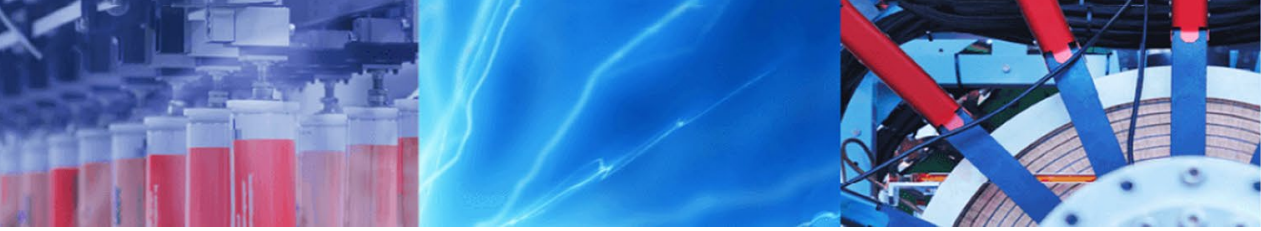

Research Article

\title{
Formation of textured Al thin film on silicone rubber to obtain superhydrophobic property
}

\author{
Masayuki Okoshi ${ }^{1}$
}

(c) Springer Nature Switzerland AG 2018

\begin{abstract}
Silicone rubber, which is widely used in electrical and biomedical applications, was modified into superhydrophobic property by the formation of a textured Al thin film on the periodic microswelling structure fabricated on silicone rubber by the $193 \mathrm{~nm}$ ArF excimer laser-induced photodissociation of silicone. The texturing of Al thin film occurred automatically during the vacuum evaporation only on the silicone rubber, compared with the cases of a slide glass or silicone resin substrate, which were observed by the scanning electron microscope and atomic force microscope. As a result, contact angle of water on the samples with 10- and 30-nm-thick, textured Al thin films was estimated to be approximately $162^{\circ}$ in both cases, compared to the sample without $\mathrm{Al}$ of approximately $155^{\circ}$. Moreover, a large air gap was remarkably formed between the Al-deposited sample and water; a droplet of colored water was successfully confined in the air gap and it was released into the water when and where need.
\end{abstract}

Keywords Silicone rubber · Periodic microswelling structure · ArF excimer laser · Al thin film · Superhydrophobic property

\section{Introduction}

Hydrophobic property, repelling water, is often useful as a property of functional materials. A silicone or fluorocarbon polymer is a promising material for realizing hydrophobic property because they are composed of $\mathrm{CH}_{3}$ or $\mathrm{CF}_{3}$ groups, respectively. These polymers are still required to have a higher hydrophobicity $[1,2]$. On the other hand, a superhydrophobic property of a plant leaf or insect is existing by nature, which is well-known as a lotus effect $[3,4]$. These are originated in a convex-concave structure of their surfaces. Thus, the formation of a convex-concave structure is effective for developing a highly hydrophobic material. For instance, micro/nanostructuring of materials, based on the laser ablation, is good processing to bring a superhydrophobic property [5-8].

A hydrophobic silicone $\left(\left[\mathrm{SiO}\left(\mathrm{CH}_{3}\right)_{2}\right]_{n}\right)$ has good properties such as electrical insulation, optical transparency, and heat-, cold- and chemicals-resistances, together with a bio-inert property. Thus, silicone is widely used for scientific and technological applications [9-11]. The peeling resistant and self-adhesive properties are also useful for industrial and biomedical uses $[12,13]$. A sealing material for light emitting diode, biochip and nanoimprint molding are a part of the industrial and practical applications. Based on those good properties, we are going to fabricate a silicone rubber-based microdevice operating in water and other solutions. To function a silicone rubber chip as a microdevice moving in water, we should apply an electric voltage to the electrodes formed on the silicone rubber chip in water. Therefore, we have considered that the surface of silicone rubber should be modified into superhydrophobic property to form an air gap layer between silicone and water [14].

In our laboratory, as another approach to obtain a superhydrophobic property using laser, we used our previous

$\triangle$ Masayuki Okoshi, okoshi@nda.ac.jp|'Department of Electrical and Electronic Engineering, National Defense Academy, Yokosuka, Kanagawa 239-8686, Japan.

SN Applied Sciences (2019) 1:133 | https://doi.org/10.1007/s42452-018-0142-4 
photochemical processing of silicone rubber to fabricate the microstructure on the surface; when a 193-nm ArF excimer laser irradiated the surface of silicone rubber, only the main chain of $\mathrm{Si}-\mathrm{O}$ bonds of silicone could be photodissociated into the lower molecules, resulting in the swelling of the laser-irradiated area [15-19]. For the microstructuring, we used microspheres made of silica glass of $2.5 \mu \mathrm{m}$ diameter, which covered the entire surface of sample during laser irradiation $[14,20]$. Very recently, we also found that the subsequent formation of a 50-nm-thick, textured $\mathrm{Al}$ thin film resulted in adding a nanostructuring effect to the microstructured silicone, showing a highly superhydrophobic property [21]. In this paper, we extend the recent experiment to the dependence of thickness of Al thin film below $50 \mathrm{~nm}$ on the texturing and obtained superhydrophobic property. The reason for texturing of Al thin film during vacuum evaporation is also discussed. In addition, a droplet of colored water is successfully confined in the air gap layer between the textured Al-deposited sample and water to be released into the water when and where need, for a demonstration.

\section{Experimental procedure}

Silica glass microspheres of approximately $2.5 \mu \mathrm{m}$ diameter (Nippon Shokubai KE-P250) of $60 \mathrm{mg}$ was dispersed in ethanol ( $99.5 \%$ purity) of $20 \mathrm{~mL}$, and the dispersed solution of approximately $10 \mu \mathrm{L}$ volume was dripped on the surface of 2-mm-thick silicone rubber of $15 \times 10 \mathrm{~mm}^{2}$. After the heated-air drying at $50^{\circ} \mathrm{C}$ for $3 \mathrm{~min}$, a single layer of the microspheres was formed on the silicone rubber, though a subsequent removal of partly overlapped microspheres was often required.

The sample was placed $80 \mathrm{~mm}$ apart from the outlet of the ArF excimer laser (Coherent COMPexPro110). The beam path was filled with argon or nitrogen gas at the flow rate of $10 \mathrm{~L} / \mathrm{min}$ to avoid strong optical absorption of oxygen molecules in air. The ArF excimer laser was conducted onto the sample surface without a lens. The single-pulse fluence of the ArF excimer laser was approximately $10 \mathrm{~mJ} / \mathrm{cm}^{2}$. The laser irradiation time was $30 \mathrm{~min}$. The pulse repetition rate was kept constant at $1 \mathrm{~Hz}$. The pulse width of the ArF excimer laser was set at approximately $20 \mathrm{~ns}$. All the laser irradiations were carried out at room temperature.

After the laser irradiation, silica glass microspheres were removed by a $1 \mathrm{wt} \% \mathrm{HF}$ chemical etching for $90 \mathrm{~s}$ and subsequent ultrasonic washing in ethanol for $10 \mathrm{~min}$, emerging the periodic microswelling structure on silicone rubber. The Al thin film was deposited on the periodic microswelling structure by a vacuum evaporation of Al wire (99.99\% purity). Thickness of the Al thin film was approximately 10 and $30 \mathrm{~nm}$, which were measured beforehand on a slide glass using a stylus profilometer (Veeco, Dektak ${ }^{3}$ ). For comparison, a flat slide glass, a flat silicone rubber and a flat silicone resin coated on a polycarbonate were used to see how the surface morphology of the deposited Al thin films changes. The surface morphology was observed by a scanning electron microscope (SEM, Phenomworld, Pro) and an atomic force microscope (AFM, Hitachi, AFM5100N).

\section{Results and discussion}

Figure 1 shows the SEM images of the periodic microswelling structure fabricated on silicone rubber surface. After the ArF excimer laser irradiation and subsequent removal of silica glass microspheres, the laser-irradiated areas underneath each microsphere clearly swelled at the regular intervals of approximately $2.5 \mu \mathrm{m}$, as shown in Fig. $1 \mathrm{a}$. The microswelling structures were also fabricated with an average of $1 \mu \mathrm{m}$ height and $1.5 \mu \mathrm{m}$ diameter. On the periodic microswelling structures, a 10- or 30-nm-thick Al thin film was deposited by the vacuum evaporation of Al wire, as shown in Fig. 1 b, $c$, respectively. The deposited Al thin films were not flat; a textured structure of Al thin film was clearly seen on both samples. For a supplemental experiment, a 10- or 30-nm-thick Au thin film was also deposited on the periodic microswelling structure; a textured structure of Au thin film was not formed in both cases.

To know the reason for the texturing of Al thin film, we deposited Al thin film on a flat silicone rubber. Figure 2 shows the SEM images of the flat silicone rubber surface with and without Al thin film. Compared to a bare silicone rubber (Fig. 2a), both the 10 and 30-nm-thick Al thin films exhibited a textured structure even on the flat silicone rubber (Fig. 2b, c). The surface roughness of the flat silicone rubbers with 10- and 30-nm-thick Al thin films was measured by the AFM, as shown in Fig. 3. The roughness was estimated to be approximately 34 and $48 \mathrm{~nm}$, respectively. Comparing to a bare silicone rubber of approximately $5.7 \mathrm{~nm}$, the surface roughness was clearly increased even after the 10-nm deposition.

We also deposited Al thin films on a flat slide glass for comparison. Under the SEM observations, as shown in Fig. 4, the Al thin film does not show a textured structure. The surface roughness taken by the AFM was to be approximately 2.6, 0.6 and $1.0 \mathrm{~nm}$ for the $0-, 10-$ and 30 -nm-thick Al thin films on flat slide glasses, respectively. In addition, a silicone resin was used for a flat substrate. As shown in Fig. 5, the Al thin film does not show a textured structure, too. The surface roughness was to be approximately 2.4 , 1.0 and $0.7 \mathrm{~nm}$ for the $0-, 10$ - and 30 -nm-thick Al thin films on flat silicone resins, respectively. Therefore, the textured 

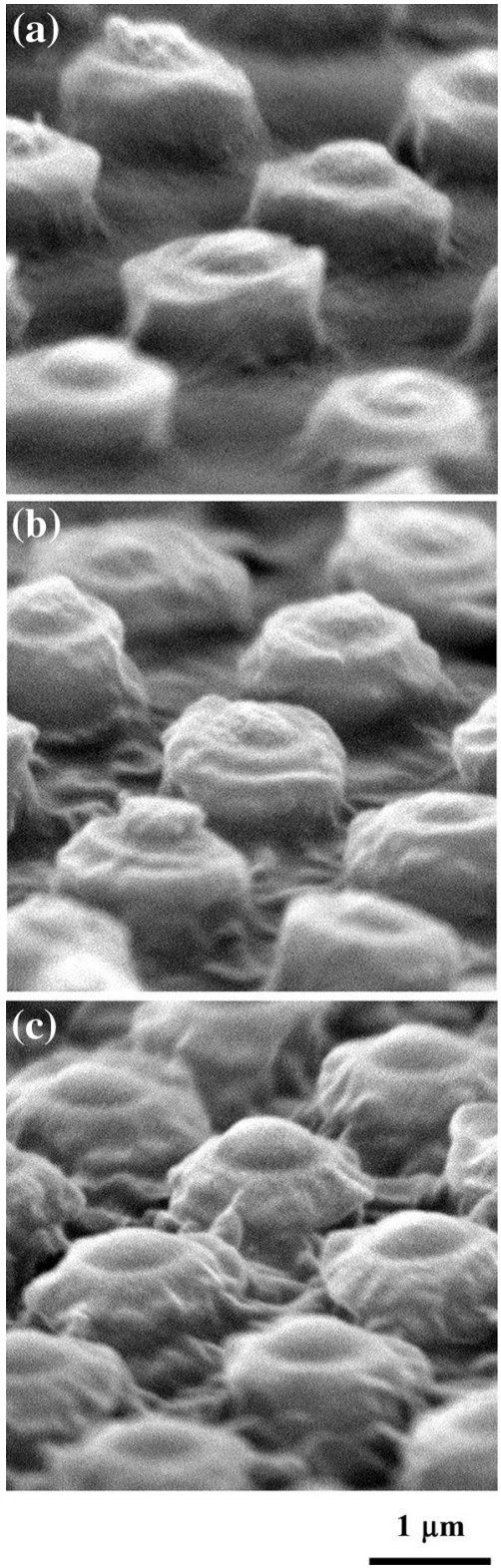

Fig. 1 SEM images of the periodic microswelling structure fabricated on silicone rubber after depositing the Al thin films with thickness of a $0 \mathrm{~nm}, \mathbf{b} 10 \mathrm{~nm}$, and $\mathbf{c} 30 \mathrm{~nm}$

structure of Al thin film could be formed only on the rubber of silicone.

As mentioned above, the surface roughness of silicone rubber, slide glass and silicone resin taken by the AFM was $5.7,2.6$ and $2.4 \mathrm{~nm}$, respectively; the roughness of silicone rubber is slightly large. Thus, one of the reasons for the texturing of Al thin film might be due to the roughened original surface of silicone rubber. However, in case of depositing an Au thin film on the flat silicone rubber, the texturing of Au thin film did not occur. Then, as another reason, we paid attention to the self-adhesive property
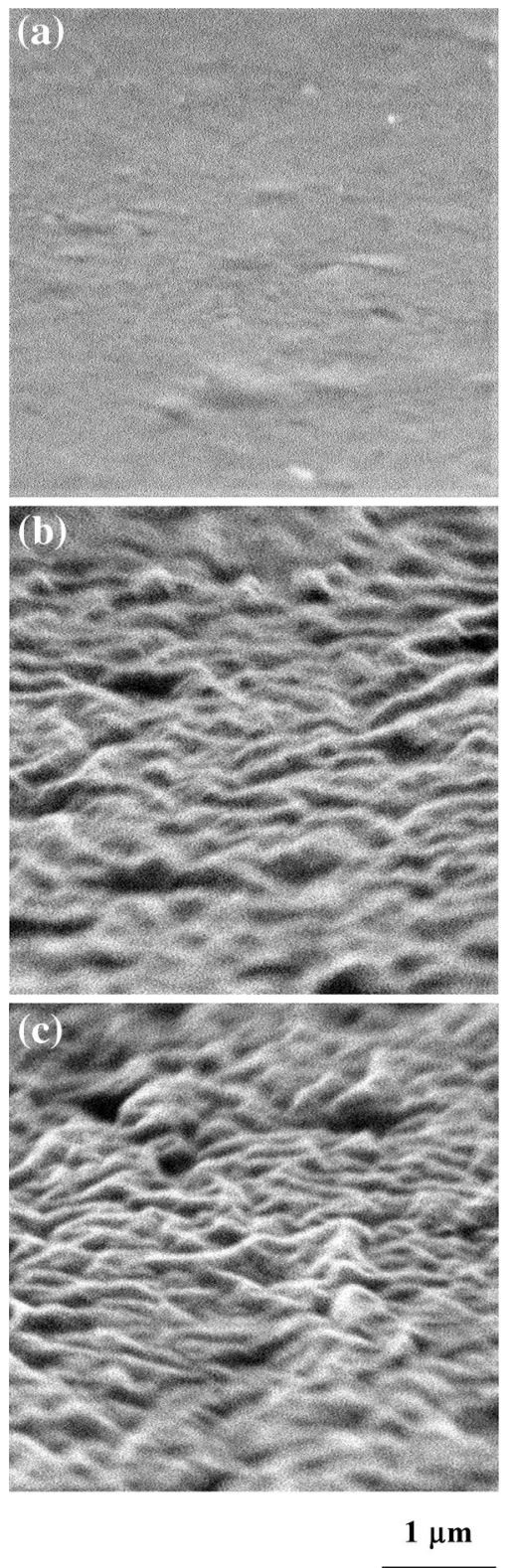

Fig. 2 SEM images of the flat silicone rubber surface after depositing the Al thin films with thickness of $\mathbf{a} 0 \mathrm{~nm}, \mathbf{b} 10 \mathrm{~nm}$, and $\mathbf{c} 30 \mathrm{~nm}$

of silicone rubber, which is originated in a low-molecular weight silicone. Generally, silicone rubber is one of the promising substrate materials for the laser induced forward transfer (LIFT) or printing method to form thin film of metals, because silicone is soft and its surface is sticky [22-24]. Thus, when the silicone rubber was used as a substrate for the vacuum evaporation, a sticking coefficient of $\mathrm{Al}$ atoms might be high to silicone rubber. Migration of the deposited Al atoms on silicone rubber would be hard, showing the textured structure.

To demonstrate a highly hydrophobic property of the textured Al deposited-silicone, the contact angle of water 

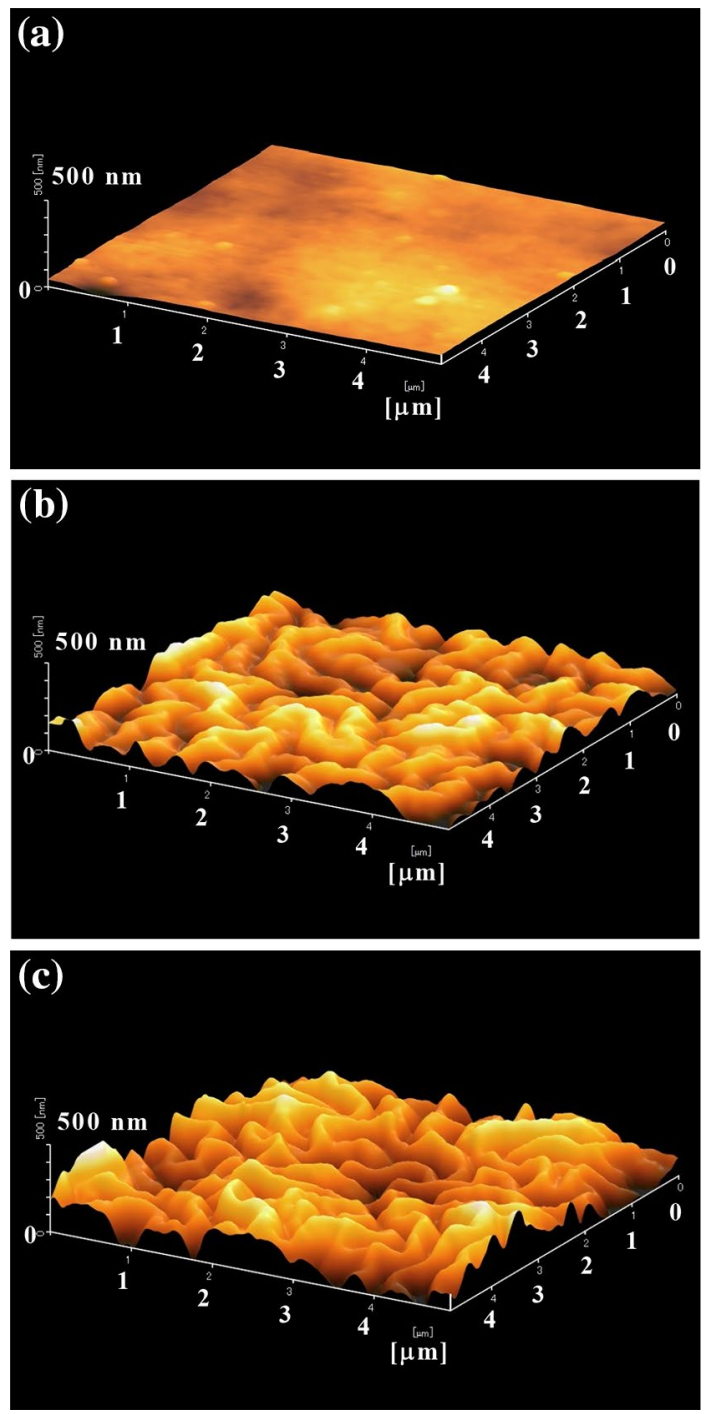

Fig. 3 AFM images of the flat silicone rubber surface after depositing the Al thin films with thickness of a $0 \mathrm{~nm}, \mathbf{b} 10 \mathrm{~nm}$, and $\mathbf{c} 30 \mathrm{~nm}$

was measured. In the measurement, a drop of water was $10 \mu \mathrm{L}$. In the case of a bare silicone rubber, the contact angle of water was approximately $90^{\circ}$ [14]. On the other hand, the periodic microswelling structure fabricated on silicone rubber showed a contact angle of approximately $155^{\circ}$, which indicates a clear superhydrophobic property. The superhydrohobic property of the sample could be explained by using the Cassie-Baxter equation [14, 25]. After depositing the 10- or 30-nm-thick Al thin film on the periodic microswelling structure, both the contact angles were successfully increased to be approximately $162^{\circ}$, as shown in Fig. 6a, b. For reference, the contact angles of water were approximately $109^{\circ}$ and $113^{\circ}$ when the 10 and 30-nm-thick Al thin films were deposited on the flat silicone rubbers, respectively. Therefore, the subsequent formation of the textured $\mathrm{Al}$ thin film resulted in adding a
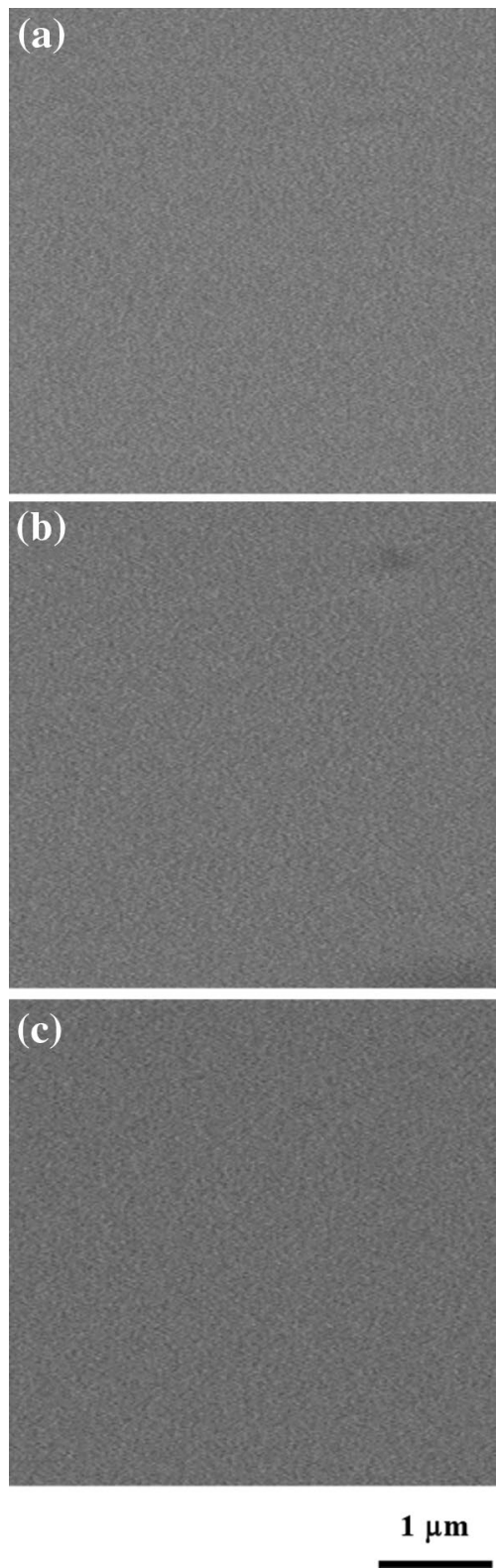

Fig. 4 SEM images of the flat slide glass surface after depositing the Al thin films with thickness of $\mathbf{a} 0 \mathrm{~nm}, \mathbf{b} 10 \mathrm{~nm}$, and $\mathbf{c} 30 \mathrm{~nm}$

nanostructuring effect to the microstructured silicone rubber; contact areas of the periodic microswelling structure with a droplet of water could be reduced.

As one of the practical demonstrations for the application to a microdevice operating in water or other solutions, the highly superhydrophobic silicone rubber depositing the 10-nm-thick, textured Al thin film was put under water, as shown in Fig. 7. In this experiment, the periodic microswelling structure was fabricated on top surface of the 2-mm-thick silicone rubber of $4 \times 4 \mathrm{~mm}^{2}$. When the sample was put under water slowly, an approximately 0.5-mm-thick, uniform air gap layer was easily formed

\section{SN Applied Sciences}



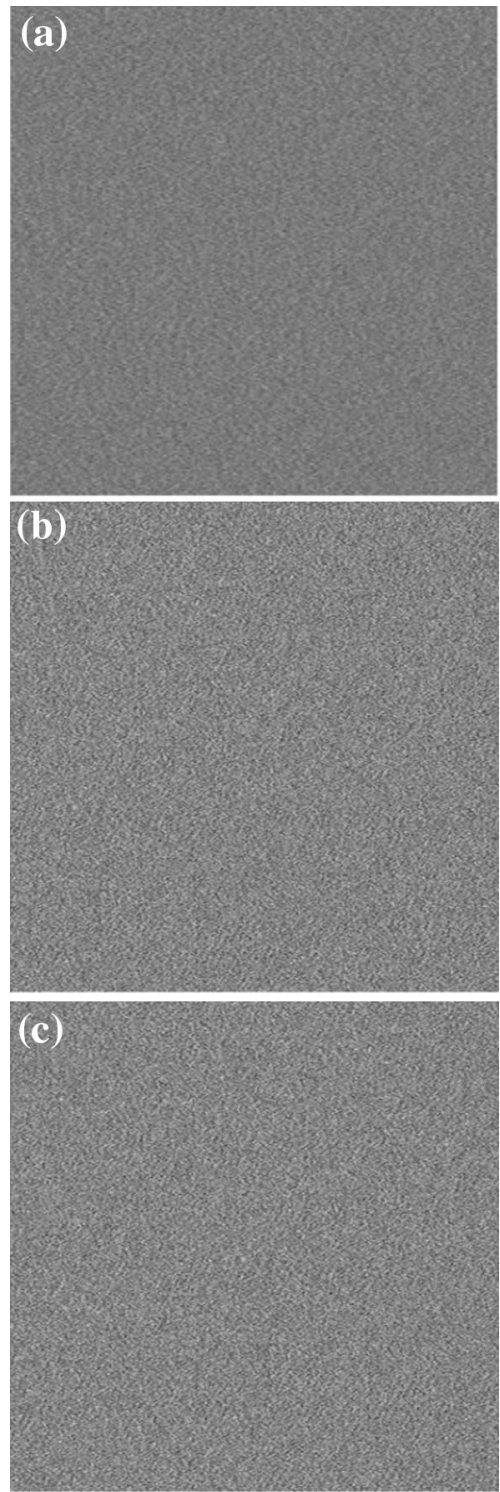

$1 \mu \mathbf{m}$

Fig. 5 SEM images of the flat silicone resin surface after depositing the Al thin films with thickness of $\mathbf{a} 0 \mathrm{~nm}, \mathbf{b} 10 \mathrm{~nm}$, and c $30 \mathrm{~nm}$

on the top surface of the sample. Then, the air gap layer could be inflated by the injection of air with a syringe [14]. Moreover, in the present work, a droplet of colored water in red was injected into the inflated air gap; the droplet was successfully confined, as shown in Fig. 7a. Then, an aspirator was used to reduce the thickness of the inflated air gap gradually (Fig. 7b). When the thickness of the air gap was reduced to the diameter of the confined droplet, the colored water was successfully released into the water (Fig. 7c). This result enables to deliver a desired liquid substance, a medicine for example, in solution and to release it into solution when and where need; it will open up the
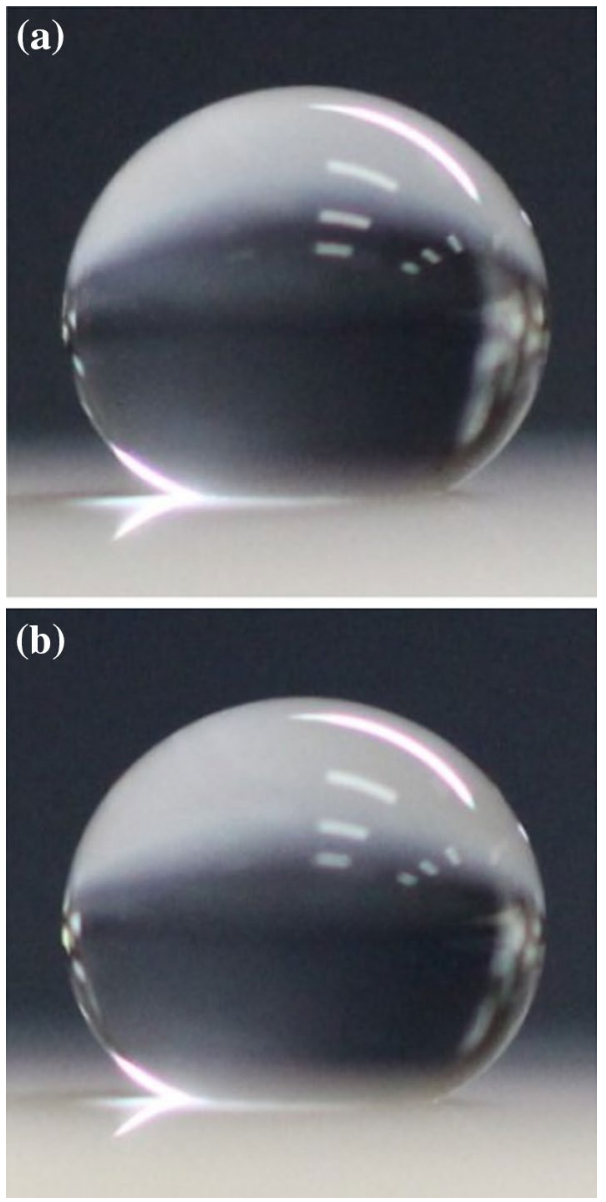

Fig. 6 Measurements of contact angle of water on the periodic microswelling structure fabricated on silicone rubber after depositing the Al thin films with thickness of $\mathbf{a} 10 \mathrm{~nm}$ and $\mathbf{b} 30 \mathrm{~nm}$

silicone rubber-based microdevice operating in water or other solutions for the application to a drug delivery system, for instance.

\section{Conclusions}

The textured Al thin film was successfully formed on the periodic microswelling structure fabricated on silicone rubber by the 193-nm ArF excimer laser-induced photodissociation of silicone to realize the highly superhydrophobic property. The texturing of Al thin film occurred automatically during the vacuum evaporation only on the silicone rubber, compared with the cases of the slide glass and silicone resin substrates. As the result, the contact angle of water on the samples with the 10and $30-\mathrm{nm}$-thick, textured $\mathrm{Al}$ thin films was estimated to be approximately $162^{\circ}$ in both cases, compared to the sample without the textured Al of approximately $155^{\circ}$. Moreover, the large air gap layer was remarkably formed 

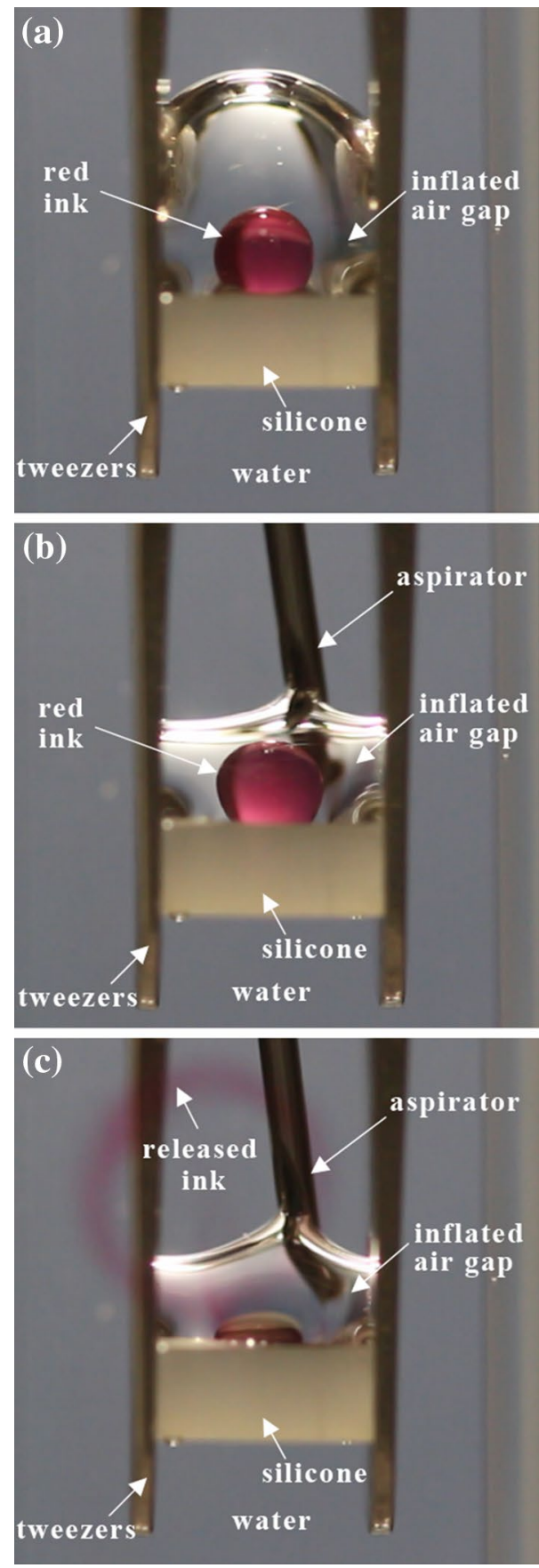

Fig. 7 Photographs of the periodic microswelling structure fabricated on silicone rubber after depositing the 30-nm-thick Al thin film in water: a a large air gap layer was inflated by the injection of air with a syringe, and a droplet of red ink was successfully confined in the air gap layer, $\mathbf{b}$ thickness of the air gap layer was decreased by an aspirator, and $\mathbf{c}$ a droplet of red ink was successfully released to the water

between the textured Al-deposited silicone and water; the droplet of colored water in red was successfully confined in the air gap to be released into the water when and where need.

\section{SN Applied Sciences}

Acknowledgements We thank Wisnu Setyo Pambudi and Ryota Matsunaga, National Defense Academy, for their valuable assistance. This work was supported by JSPS KAKENHI Grant Number JP18K04790.

\section{Compliance with ethical standards}

Conflict of interest The corresponding author states that there is no conflict of interest.

\section{References}

1. Nakajima A, Hashimoto K, Watanabe T (2001) Recent studies on super-hydrophobic films. Monats Chem 132:31-41

2. Emelyanenko AM, Boinovich LB, Bezdomnikov AA, Chulkova EV, Emelyanenko KA (2017) Reinforced superhydrophobic coating on silicone rubber for longstanding anti-icing performance in severe conditions. Appl Mater Interfaces 9:24210-24219

3. Barthlott W, Neinhuis C (1997) Purity of the sacred lotus, or escape from contamination in biological surfaces. Planta 202:1-8

4. Gao X, Yan X, Yao X, Xu L, Zhang K, Zhang J, Yang B, Jiang L (2007) The dry-style antifogging properties of mosquito compound eyes and artificial analogues prepared by soft lithography. Adv Mater 19:2213-2217

5. Ranella A, Barberoglou M, Bakogianni S, Fotakis C, Stratakis E (2010) Tuning cell adhesion by controlling the roughness and wettability of 3D micro/nano silicon structures. Acta Biomater 6:2711-2720

6. Cardoso MR, Tribuzi V, Balogh DT, Misoguti L, Mendonca CR (2011) Laser microstructuring for fabricating superhydrophobic polymeric surfaces. Appl Surf Sci 257:3281-3284

7. Vorobyev AY, Guo C (2015) Multifunctional surfaces produced by femtosecond laser pulses. J Appl Phys 117:033103

8. Ta VD, Dunn A, Wasley TJ, Li J, Kay RW, Stringer J, Smith PJ, Esenturk E, Connaughton C, Shephard JD (2016) Laser textured superhydrophobic surfaces and their applications for homogeneous spot deposition. Appl Surf Sci 365:153-159

9. Ganachaud F, Boileau S, Boury B (2008) Silicone based polymers. Springer, Berlin

10. Li M, Neoh KG, Xu LQ, Wang R, Kang E-T, Lau T, Olszyna DP, Chiong E (2012) Surface modification of silicone for biomedical applications requiring long-term antibacterial, antifouling, and hemocompatible properties. Langmuir 28:16408-16422

11. Liu P, Chen Q, Yuan B, Chen M, Wu S, Lin S, Shen J (2013) Facile surface modification of silicone rubber with zwitterionic polymers for improving blood compatibility. Mater Sci Eng C 33:3865-3874

12. Owen MJ, Dvornic PR (2012) Silicone surface science. Springer, Berlin

13. Wilbur JL, Kumar A, Kim E, Whitesides GM (1994) Microfabrication by microcontact printing of self-assembled monolayers. Adv Mater 6:600-604

14. Okoshi M, Pambudi WS (2016) Fabrication of superhydrophobic silicone rubber by ArF-excimer-laser-induced microstructuring for repelling water in water. Appl Phys Express 9:112701

15. Takao H, Okoshi $\mathrm{M}$, Inoue $\mathrm{N}$ (2002) Fabrication of $\mathrm{SiO}_{2}$-humps on silicone rubber using $F_{2}$ laser. Jpn J Appl Phys 41:L1088-L1089

16. Okoshi M, Kimura T, Takao H, Inoue N, Yamashita T (2004) Photochemical modification of silicone films using $F_{2}$ laser for selective chemical etching. Jpn J Appl Phys 43:3438-3442

17. Takao H, Okoshi M, Inoue N (2004) Swelling and modification of silicone surface by $F_{2}$ laser irradiation. Appl Phys $A$ 79:1571-1574 
18. Takao H, Okoshi M, Miyagami H, Inoue N (2004) Fabrication of $\mathrm{SiO}_{2}$ microlenses on silicone rubber using a vacuum-ultraviolet $F_{2}$ laser. IEEE J Sel Top Quant 10:1426-1429

19. Takao H, Miyagami H, Okoshi M, Inoue N (2005) Microlenses fabricated on silicone rubber using an $\mathrm{F}_{2}$ laser. Jpn J Appl Phys 44:1808-1811

20. Okoshi M, Awaihara Y, Yamashita T, Inoue N (2014) $\mathrm{F}_{2}$-laser-induced micro/nanostructuring and surface modification of iron thin film to realize hydrophobic and corrosion resistant. Jpn J Appl Phys 53:112701

21. Okoshi M (2018) Fabrication of superhydrophobic silicone rubber operating in water. Appl Phys Express 11:101801
22. Tsuboi Y, Furuhata Y, Kitamura N (2007) Laser-induced forward transfer of luciferase onto a PDMS microchip as an ATP sensor. Appl Surf Sci 253:8422-8427

23. Narazaki A, Sato T, Niino H (2018) Laser-induced forward transfer with optical stamp for high-quality film printing. Proc SPIE 10519:051900

24. Lee HM, Choi S-Y, Kim KT, Yun J-Y, Jung DS, Park SB (2011) A novel solution-stamping process for preparation of a highly conductive aluminum thin film. Adv Mater 23:5524-5528

25. Cassie ABD, Baxter S (1944) Wettability of porous surfaces. Trans Faraday Soc 40:546-551 